\title{
Assessing Conformer Energies: \\ Machine Learning vs Conventional Quantum Chemistry
}

\& Dakota Folmsbee and Geoffrey Hutchison

\section{Motivation}

Understanding and predicting thermodynamically accessible conformations is a key task as the number of possible minima increase exponentially for each rotatable bond. This becomes expensive for wavefunction and density functional first principles methods leading to a desire of a fast and accurate method, such as machine learning (ML), to be used as a surrogate for these time-consuming calculations.

\section{Methodology}

- Conformer dataset consists of $~ 6000$ DLPNO$\operatorname{CCSD}(\mathrm{T})$ single points of $\sim 700$ small molecules with up to 10 conformers each containing atoms of $\mathrm{H}, \mathrm{C}, \mathrm{N}, \mathrm{O}, \mathrm{F}, \mathrm{P}, \mathrm{S}, \mathrm{Cl}$, and/or $\mathrm{Br}$.

- Evaluated 39 methods including dispersion and non-dispersion corrected DFT and normalized ML methods.

\section{Performance}

- RI-MP2 and DFT dispersion methods perform best at the cost of time while $M L$ and semiempirical methods look to be an even ground between accuracy and time.

- Most methods are dominated by random errors as measured with $\mathrm{R}^{2}$.

- A few force field and ML methods show increased MARE from systematic errors.

\section{Thanks}

This research was supported by the NSF and the University of Pittsburgh Center for Research Computing. We also thank Olexandr Isayev and Justin Smith for access to the ANI-2x model.

\section{Across 10 Conformers}

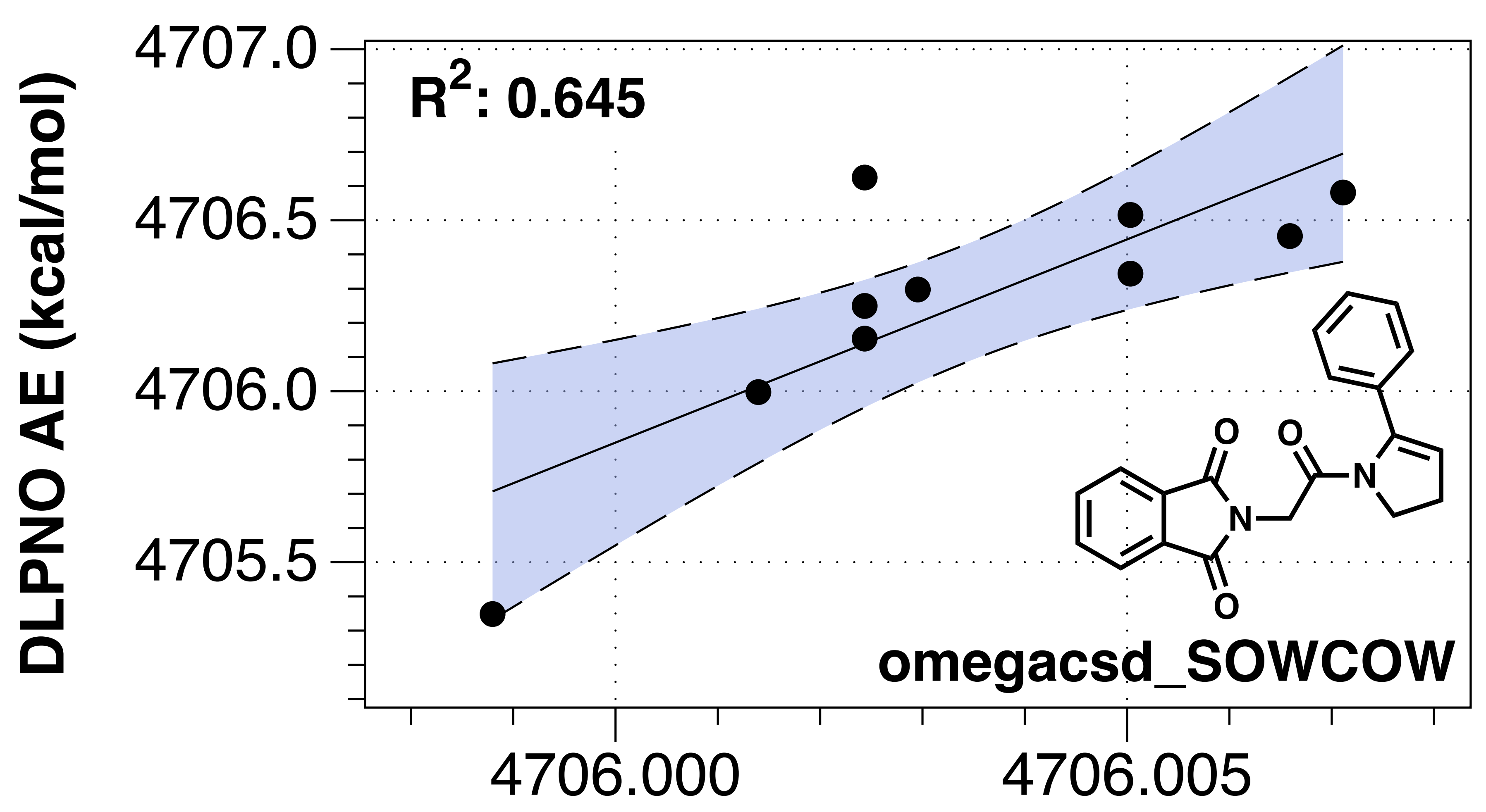

ANI-1ccx Energy (kcal/mol)

\section{Across 700 Molecules}
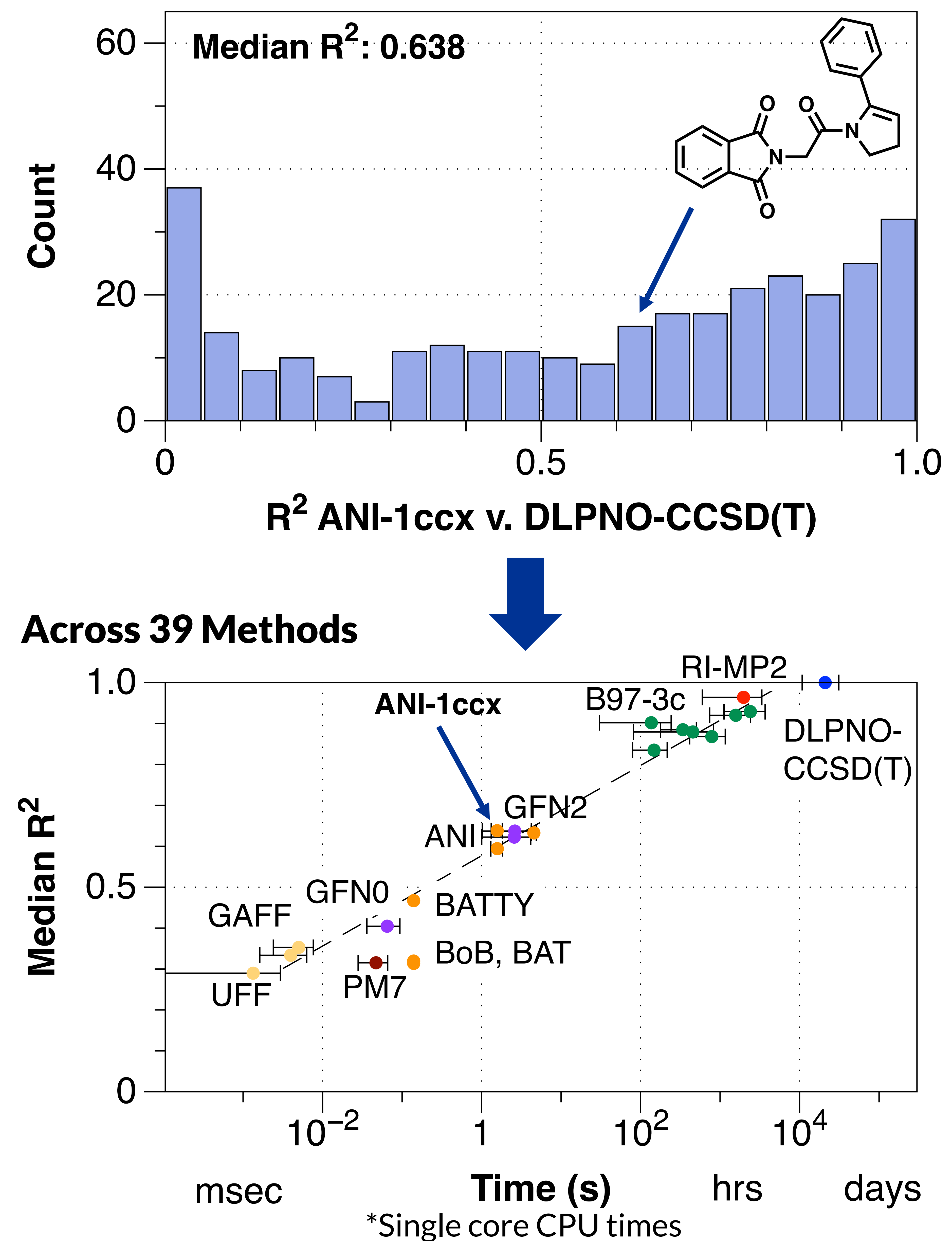

ML methods show promise with comparable accuracy to semiempirical methods and can be accelerated on GPUs. We expect these methods to provide increasing accuracy. 